\title{
THE RADIATION SAFETY INTERLOCK SYSTEM FOR TOP-UP MODE OPERATION AT NSRRC
}

\author{
C. R. Chen, F. D. Chang, J. Liu, J. P. Wang,R. J. Sheu, S. P. Kao \\ National Synchrotron Radiation Research Center \\ 101 Hsin-Ann Road, Hsinchu Science Park, Hsinchu 300, Taiwan, R.O.C.
}

\begin{abstract}
The radiation safety interlock systems of the National Synchrotron Radiation Research Center (NSRRC) have been operated for more than a decade. Some modification actions have been implemented in the past to perfect the safe operation. The overall performance of this interlock system is reliable and satisfactory. The machine and its interlock system were originally designed to operate at decay mode. During decay mode injection, the safety shutters of beamlines are closed and the radiation loss is contained inside accelerator tunnel. Recently, some modification programs to change operation mode from original decay mode to top-up mode at NSRRC have initiated. For users at experimental area, the radiation dose resulted from top-up refilled injections when safety shutters of beamlines are opened will dominate. To ensure personnel radiation safety for top-up operation, the safety interlock upgrade and other action plans will be implemented. This paper will summarize the original design logic of the Safety Interlock System (SIS), its historical modifications and the new design logic to ensure radiation safety for top-up operation.
\end{abstract}

\section{INTRODUCTION}

NSRRC has a third generation synchrotron light source that consists of a $50 \mathrm{MeV}$ LINAC, a $1.5 \mathrm{GeV}$ booster synchrotron and a six-fold symmetrical electron storage ring with $120 \mathrm{~m}$ circumference. The layout of the accelerator facilities is shown in Figure 1. Formerly, each shift takes several minutes to fill the ring with full store current of $200 \mathrm{~mA}$ and then follows by a beam decay period of about 8 hours for user's experiments. This kind of operation is defined as decay mode. The radiation safety interlock system was designed to protect personnel from exposure to radiation present when the facilities started operating at 1992. To take the advantages of reducing thermal effect of beamline optical elements and giving more stable and intense photon beams, NSRRC has set a goal to start the commissioning of top-up operation at the beginning of 2006. Top-up operation is a newly developed operation mode for synchrotron facilities. The store current will be maintained at near full current by using frequent injections, while shutters of beamlines are open and photon beams are delivered to end stations without refill breaks. Due to frequent injections, keeping at high store current and especially opening beamline safety shutters during injections, the radiation dose of top-up operation will surely be higher than that from present decay mode. The injection efficiency from BTS (Booster to storage Transport
System) to storage ring is an important factor and could vary significantly. Adopting current average injection efficiency of $30 \%$ and using the method described in Ref. (7), the maximum annual dose for a user working at beamline area at NSRRC is $1.47 \mathrm{mSv}$ for $200 \mathrm{~mA}$ decay mode and $4.60 \mathrm{mSv}$ for $200 \mathrm{~mA}$ top-up mode, respectively. We adopt a much stringent dose limit (2 $\mathrm{mSv}$ per year) for radiation workers at NSRRC than the regulatory requirement $(20 \mathrm{mSv}$ per year) set by the Atomic Energy Council of Taiwan. In order to keep the present dose limit of $2 \mathrm{mSv} / \mathrm{y}$ for radiation workers at the NSRRC after switching to top-up operation, a series of radiation safety analyses including calculations and measurements have been performed. Some action plans such as upgrading the shielding of the injection section, enlarging the exclusion zones of the straight section beamlines, installing the hall probe at the end of the injection section, installing an interlocked radiation monitoring system for each ID (Insertion Device) beamline and improving the injection efficiency for topup operation are also scheduled in the near future. To prevent the mistakes of operators and to protect the workers from accidental high exposure, the related radiation safety interlock system for top-up mode will also be implemented.

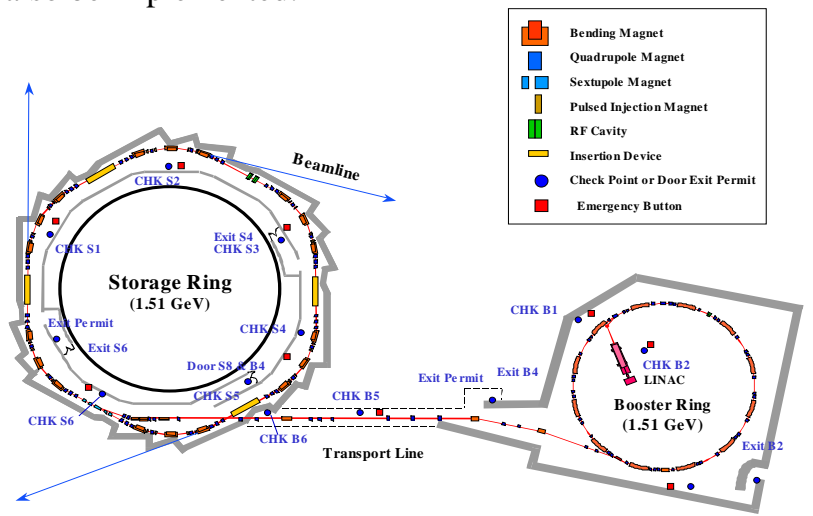

Figure 1: The simplified layout of NSRRC.

\section{OVERVIEW OF THE SAFETY INTERLOCK SYSTEM}

The interlock systems at NSRRC have been operated smoothly in decay mode since 1994. Just like the other synchrotron radiation research facilities, the interlock systems are important safeguards to make sure personnel safety during machine operation. The main characteristics are independent, reliable, fail-safe, testable, unrestrictive and redundant. The controlled area of NSRRC is divided into several zones with different levels of access 
authorizations: free, restricted and prohibited area. The status indicators show four conditions, Reset in Green, Search in Yellow, Ready in Red and Injection in flash Red light. When the machine is shutdown, the whole area is free for access. The experimental hall is a controlled zone during accelerator operation where personnel radiation monitoring with TLD (Thermo Luminescent Dosimeters) and area radiation monitoring with active detectors. The integrity of access system is a key element for safe accelerator operation. It must be highly robust in order to ensure radiation safety. As shown in Figure 2, the SIS at NSRRC consists of four subsystems. Each subsystem operates separately and is integrated to make up a complete SIS. The upper part of SIS is the Access Control System (ACS) of booster and storage ring. Access control, automatic-broadcasting, search confirmation, injection warning, emergency shut down are the basic functions of the ACS. The middle parts of SIS are Fronted-end Interlock System (FIS), Beamline Enable Controller (BEC), and the Top-up mode Interlock System (TIS). The BEC controls the enable mechanism of the main Hutch Interlock System (HIS). The TIS is a newly developed subsystem that is built on relays. The lower part of SIS is HIS. Figure 3 shown the layout of a typical beamline, its key components and associated subsystems of the SIS. Each subsystem consisting of relays and indicators is installed in a separate rack. The ACS, TIS and HIS are relay-based systems. The FIS, BEC and several newly updated HIS are controlled by Programmable Logical Controller (PLC).

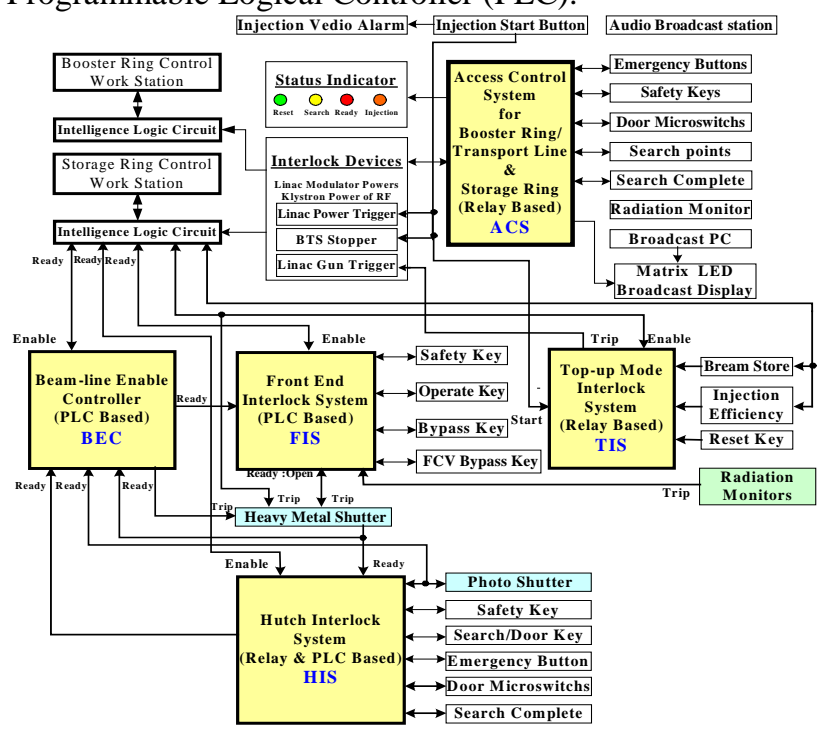

Figure 2: The main flow diagram of the safety interlock system showing the overall safety system and the related subsystems at NSRRC.

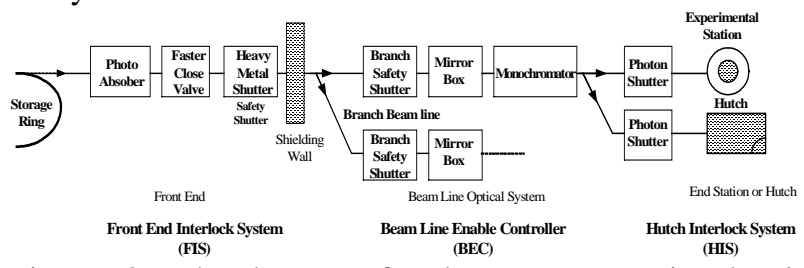

Figure 3: The layout of subsystems associated with beamline operations.

\section{THE TOP-UP MODE INTERLOCK SYSTEM}

To exploit the advantages of constant heat-load and intense photon flux, we began a two-year project to switch to top-up operation from 2004. This project includes hardware and software upgrades of the machine as well as radiation safety enhancement. The final goal of this upgrade is to keep radiation dose of the workers and users at NSRRC below the design goal of $2 \mathrm{mSv} / \mathrm{y}$. Before reaching this goal, there are several measures to ensure radiation safety. Above all, the injection efficiency is most important. To make sure the injection efficiency is qualified and the radiation level is satisfied, the existing radiation interlock logic must be incorporated into the SIS and new logics must be added to prevent dipole failure and injection difficulty as well as unexpected radiation exposure. The top-up operation at NSRRC will be initiated under the protection of TIS. The logic block diagram of the TIS is shown in Figure 4. The principle signals for input and output are described below.

\section{The Signals to Start and Stop the Injection}

The Heavy Metal Shutter (HMS) is an important safety device to block the radiation produced inside the tunnel from passing through the beamline hole on the ratchet wall during injection. It is a policy of NSRRC to close HMS during fresh injection (0 to 200mA) either decay mode or top-up mode. HMS is also closed for decay mode refill injection (120 to $200 \mathrm{~mA}$ ). However, it is open during top-up refilled injection that occurs every two minutes while beam current may decay from $200 \mathrm{~mA}$ to $199 \mathrm{~mA}$. This difference will require a signal to start and keep HMS open during top-up refilled injection.

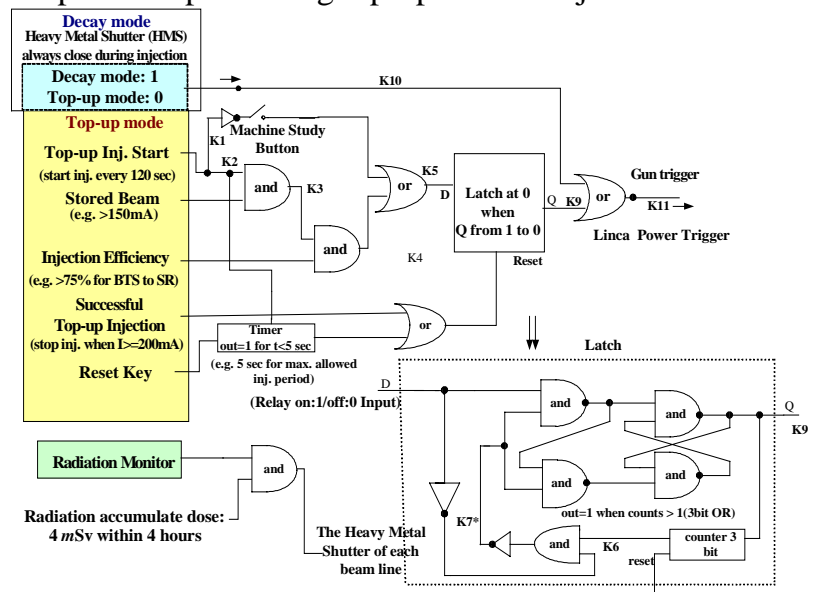

Figure 4: The logic diagram of top up mode interlock system.

\section{Conditions to Proceed to the Top Up Mode Injection}

There are several requirements to radiation impact to users, including the existence of stored beam (e.g. I $>150$ $\mathrm{mA}$ ) and qualified efficiency (e.g. > 75\%). Top-up operation can proceed only as these two conditions are true.

\section{Interlock Devices}


Once a TIS fault happens, i.e. there are conditions unable to fulfil top up mode requirements, a signal will be sent to ACS to terminate the top-up refilled injection. The ACS will turn off the injection related devices, such as the booster RF (Radio Frequency) gun trigger and power supply trigger of the booster ring, but will not trip or dump the store beam. In this situation, top-up injection is prohibited while decay mode injection is still allowed. A manual reset process by safety person must be conducted before top-up injection can resume.

\section{Radiation Monitoring}

Six sets of radiation monitoring stations will be installed at the accessible area of six straight section beamlines. Interlock logic will also be added to our safety interlock system to ensure that accumulated dose from each monitor will not exceed $4 \mu \mathrm{Sv}$ within 4 hours. If the accumulate dose exceeds the setting, the HMS will be closed for the remaining of the 4 hours period. This interlock logic ensures that annual personnel dose will not exceed the limit of $2 \mathrm{mSv}$.

\section{IMPLEMENT OF THE TOP-UP MODE INTERLOCK SYSTEM}

The relay based interlock system is stable and reliable but is complicated in implementation and wiring. For improving and taking the merits of advantage of electronic technology, the core of the interlock system is gradually switching to PLC in recent years. The advantages of PLC are compact, effort saving, easy programming, and easy modification. Both digital and analogy I/O (Input and Output) signals can be processed by PLC system. However, there may be some potential defects for PLC system according to our experimental experience. Because the logic of PLC is established by programming that is easy to revise, careful verification of programming and intensive debug have become very important. Since there are microprocessor and system clock inside the package of PLC, it is not a pure hardwire but a firmware instead. The suspension and sluggishness are possible defects, because the reliability of PLC is not so crucially tested as relay-based circuit. If the volume of necessary support components of interlock system such as indicators, switches, output connectors, and others are considered, the advantage of PLC being small-scale will not be true compare to the relay-based circuit. New TIS uses the relays because its scale of circuit is small. From the viewpoints of volume and reliability, PLC is not necessarily the best choice. For the design of interlock system we have to find the optimum balance between safety, reliability, volume, implement and cost of the system.

\section{SUMMARY}

The proposed top-up operation that opens safety shutter during injection will introduce additional beam loss scenarios, especially when the injection efficiency is poor. Before top-up operation can benefit users with better quality of synchrotron light in the future, safety upgrades and detailed dose assessments have to be completed to ensure radiation safety. Obviously, without any machine improvement or shielding upgrade, top-up operation will not be allowed under present dose limit of the NSRRC. Safety upgrades for accelerator and beamlines have to be completed to ensure radiation safety, such as the improvement of injection efficiency, increasing the local shielding of injection section, installing hall probes or permanent magnets at the end of the injection section to prevent mis-steered electrons from streaming into beamline and adding interlocked radiation monitoring system at each straight section beamline. The TIS installed at control room is another important safety device. Although we have designed this system, there are still several input parameters need to be clearly defined with the helps from machine physicists. We are confident that the operation performance of TIS will surely give us some positive feedback when top-up commissioning starts at the end of this year. The successful implementation and operation of this system will ensure operation safety of NSRRC when top-up operation facilitates users to explore new era.

\section{REFERENCES}

[1] The design guidelines for the beamlines safety interlock system of NSRRC, Beamline Safety Committee, 1999.05, (in Chinese).

[2] T.F. Lin, J.P. Wang, The Safety Interlock System of Synchrotron Radiation Research Center, Particle Accelerator Conference and International Conference on High Energy Accelerators, 1995.

[3] A.Vascotto, D. Bulfone, F. Giacuzzo, S Grulja, M. Lonza, G. Tromba, Sincrotrone Trieste, Italy, A.Mazzoli, M. Salvador, FASI TECH srl, Pordenone, Italy, The Upgrade ELETTRA Access Control System. International conference on Accelerator and Large Experimental Physics Control Systems, 1999, Trieste, Italy.

[4] Emery L. and Borland M. Top-up operation experience at the advanced photon source. In: Proc. 1999 Particle Accelerator Conf., New York, March 29-April 2 1999, pp. 200-202.

[5] Lüudeke A. and Muñoz M. Top-up operation experience at the Swiss light source. In: Proc. European Particle Accelerator Conf. 2002, Paris, June 3-7, 2002. pp. 721-723.

[6] Job P. K. Experiences in radiation safety during the topup operation at the advanced photon source. In: Proc. 2nd Int. Workshop on Radiation Safety at Synchrotron Radiation Sources, Grenoble, October 17-18, 2002.

[7] Sheu R. J., Wang J. P., Liu J., Chen C. R., and Chang F. D., Jiang S.H., 2004. Radiation safety impact for top-up operation at the NSRRC. Proceedings of the $10^{\text {th }}$ International Conference on Radiation Shielding and $13^{\text {th }}$ Topical Meeting on Radiation Protection and Shielding, Funchal, Madeira Island, Portugal (to be published in Radiation Protection Dosimetry), 